LAWRENCE LIVERMORE NAT IO N A L LABORATORY

\section{Adapting high-resolution speckle imaging to moving targets and platforms}

C. J. Carrano, J. M. Brase

February 5, 2004

SPIE Defense and Security Symposium Orlando, FL, United States April 12, 2004 through April 16, 2004 
This document was prepared as an account of work sponsored by an agency of the United States Government. Neither the United States Government nor the University of California nor any of their employees, makes any warranty, express or implied, or assumes any legal liability or responsibility for the accuracy, completeness, or usefulness of any information, apparatus, product, or process disclosed, or represents that its use would not infringe privately owned rights. Reference herein to any specific commercial product, process, or service by trade name, trademark, manufacturer, or otherwise, does not necessarily constitute or imply its endorsement, recommendation, or favoring by the United States Government or the University of California. The views and opinions of authors expressed herein do not necessarily state or reflect those of the United States Government or the University of California, and shall not be used for advertising or product endorsement purposes. 


\title{
Adapting high-resolution speckle imaging to moving targets and platforms
}

\author{
C. J. Carrano and J. M. Brase \\ Lawrence Livermore National Laboratory, 7000 East Avenue, Livermore, CA 94551
}

\begin{abstract}
High-resolution surveillance imaging with apertures greater than a few inches over horizontal or slant paths at optical or infrared wavelengths will typically be limited by atmospheric aberrations. With static targets and static platforms, we have previously demonstrated near-diffraction limited imaging of various targets including personnel and vehicles over horizontal and slant paths ranging from less than a kilometer to many tens of kilometers using adaptations to bispectral speckle imaging techniques ${ }^{1,2,3}$. Nominally, these image processing methods require the target to be static with respect to its background during the data acquisition since multiple frames are required. To obtain a sufficient number of frames and also to allow the atmosphere to decorrelate between frames, data acquisition times on the order of one second are needed. Modifications to the original imaging algorithm will be needed to deal with situations where there is relative target to background motion. In this paper, we present an extension of these imaging techniques to accommodate mobile platforms and moving targets.
\end{abstract}

\section{INTRODUCTION}

High-resolution imaging over long horizontal or slant paths is a problematic task due to atmospheric turbulence. To increase imaging resolution, increased aperture sizes are needed (or shorter wavelengths). Ideally with no atmosphere, the best optical resolution is $\lambda / D$, where $\lambda$ is the wavelength and $\mathrm{D}$ is the aperture diameter. But, if $\mathrm{D}$ gets large enough, the resolution actually becomes limited by the atmosphere, or $\lambda / \mathrm{r}_{0}$, where $\mathrm{r}_{0}$ is the Fried atmospheric coherence length ${ }^{4}$. The ratio of $\mathrm{D} / \mathrm{r}_{0}$ is then a measure of how much atmospheric blurring there is compared to the diffraction limit. Over horizontal or low slant paths, this $\mathrm{r}_{0}$ value is often quite small, typically $0.5 \mathrm{~cm}$ to $2 \mathrm{~cm}$ at visible wavelengths, meaning that aperture sizes greater than $r_{0}$ will not provide increased resolution unless something is done to mitigate the atmospheric turbulence.

We have demonstrated via many experiments the usefulness of sub-field speckle image processing to compensate atmospheric blurring effects ${ }^{1,2,3}$. Because the algorithm requires multiple frames of imagery, where the primary variation from image to image is the ever changing atmospheric distortion, a stationary imaging platform and target is required. We briefly discuss the basic algorithm and static target results in Section 2. Strictly speaking, motion of the imaging platform alone will not prevent the algorithm from working. If the camera is on a suitably stabilized platform, it should be able to keep the target of interest in its field of view. Likewise, if the camera operates at video frame rates, meaning that data acquisition only requires a second or two, large changes in viewing angle should not occur. Some global motion or shaking of the entire image is acceptable and mitigated via a frame by frame image registration which is always performed early in the processing.

The primary problem will occur when the target being imaged is moving with respect to its background, such as with a vehicle driving down a road. In order to provide a high-resolution imaging capability for situations where there is relative motion between the target and background, it becomes necessary to perform additional pre-processing steps prior to applying the sub-field speckle processing technique. These steps are explained, along with example results in Section 3.

\section{METHOD AND RESULTS WITH STATIC PLATFORM AND TARGETS}

\subsection{Sub-field speckle image processing method overview}

In Ref. 1 we describe the details and rationale of the sub-field image processing using bispectral speckle-imaging techniques. The primary processing steps are summarized in Figure 1. After obtaining the raw camera images, we remove sensor artifacts by flat-fielding, that is, dividing each frame by a time average of reference flat-field frames. 
The frames are then registered to each other by applying shifts calculated from frame to frame cross-correlation. The large image data cube is next split up into sub-fields, which are overlapped by $50 \%$. A typical tile size that works well in many situations is $128 \times 128$ or $256 \times 256$ pixels. The sub-fields are then apodized and reconstructed using bispectral speckle imaging algorithms ${ }^{5}$. The user defined apodization consists of a flat region in the middle with a Hanning window taper at the edges. Typical apodization widths are $60 \%$ of the window size, where the center $60 \%$ is flat and the outer $20 \%$ on each side is tapered. Finally, the processed tiles are reassembled to form the full sized image.

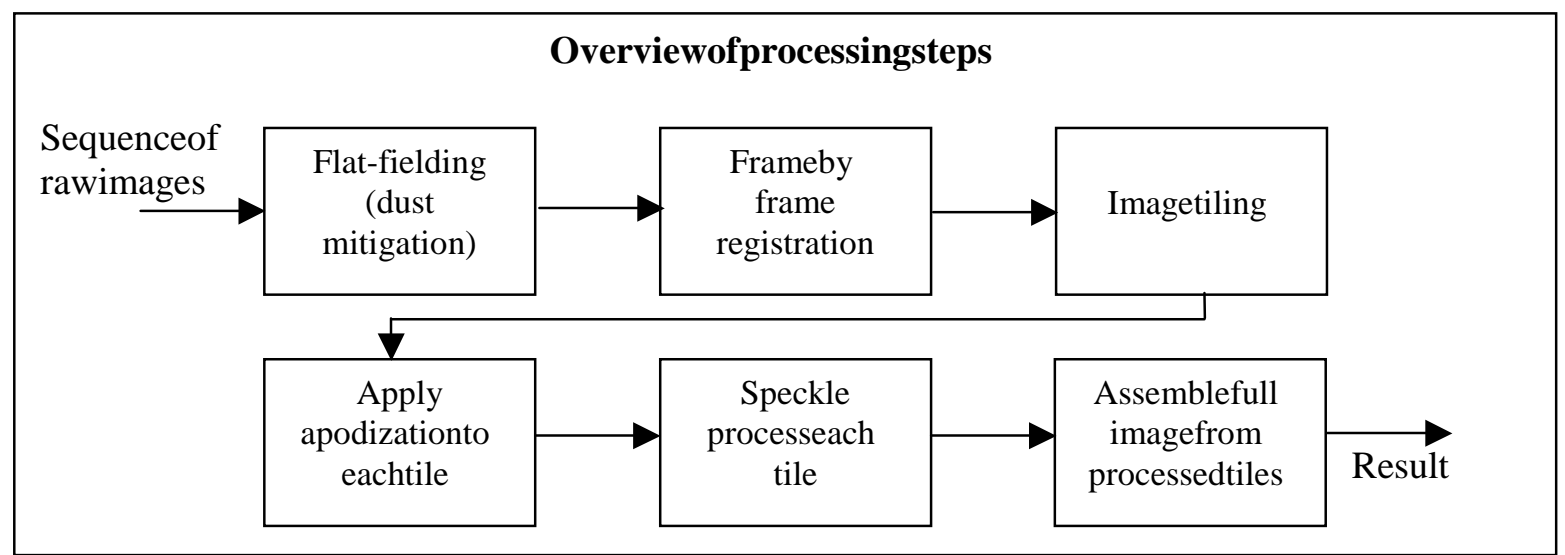

Figure 1: Block diagram overview of processing steps for producing enhanced images of extended scene, horizontal path imagery via speckle processing.

\subsection{Experimental results}

Our equipment consisted of an 8-inch Celestron telescope, a 3x Barlow lens as needed, a high-resolution CCD camera capable of short exposures and a computer for data acquisition and processing. Pixel sizes mapped to the object plane vary from one half the diffraction limit (Nyquist sampling) for short ranges to about three times the diffraction limit for longer ranges. Exposure times typically range from 1 to 20 milliseconds depending on illumination levels.

\subsubsection{Stationary imaging of personnel}

The first two imaging results we show were obtained from a low hillside vantage point approximately 500 feet above the road below. In Figure 2 we show before and after images of a person from $1.3 \mathrm{~km}$ range holding a box with $1.6 \mathrm{~cm}$ tall letters spelling the word "MAGELLAN" on it. In the raw image on the left, the face is blurry and words unreadable, but in the processed image on the right, the face is recognizable and we can clearly read the word. Note that the diffraction limit for this case is approximately $0.4 \mathrm{~cm}$.

In Figure 3, we show before and after images of personnel and a resolution target but now from $3.3 \mathrm{~km}$ away. The speckle processing gives a near-diffraction limited reconstruction of the scene as evidenced by the facial and hand details on the order of $1 \mathrm{~cm}$ in size now observable.

To test whether the speckle processing technique would work under low light conditions, we acquired imagery at sunset using a Generation III intensifier in the form of a pocketscope placed between the telescope and the CCD camera. Before and after images of personnel and a car imaged from a $1.5 \mathrm{~km}$ distance over a horizontal path at sunset are shown in Figure 4. Much of the noisiness in the image is due to photon noise and the use of the intensifier. The speckle processed image gives greater detail in all aspects of the scene; most notable is the clearly readable license plate. 


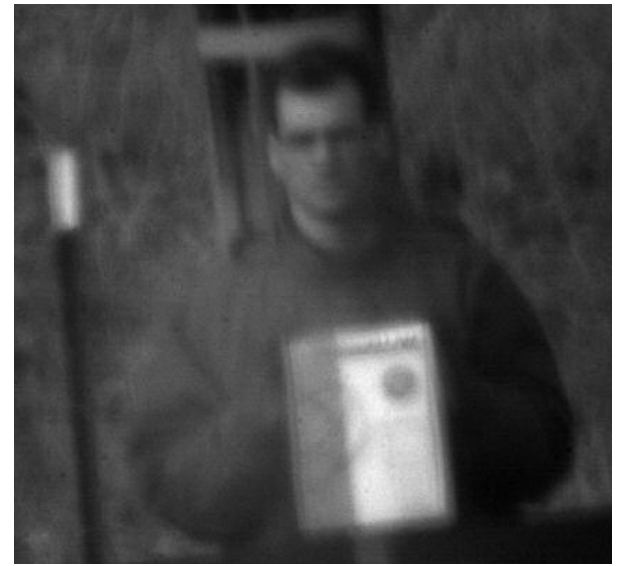

2a: Blurry, unprocessed raw image

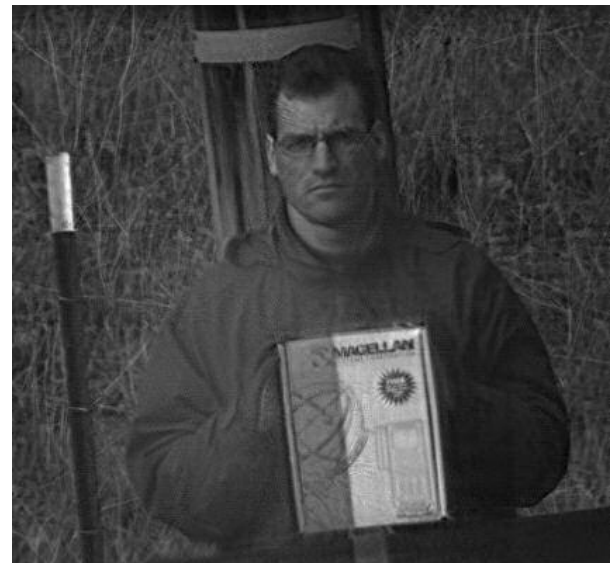

$2 \mathrm{~b}$. Speckle processed result from 100 raw frames

Figure 2: Personnel imaged at a $1.3 \mathrm{~km}$ distance using a 10 millisecond exposure time.

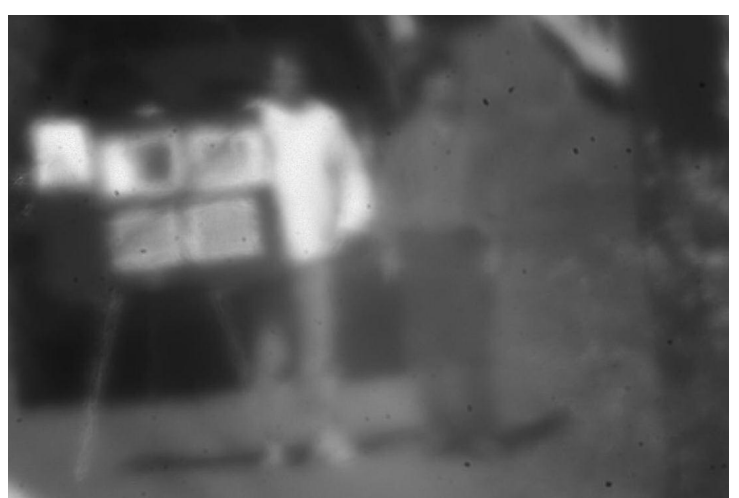

3a: Blurry, unprocessed raw image

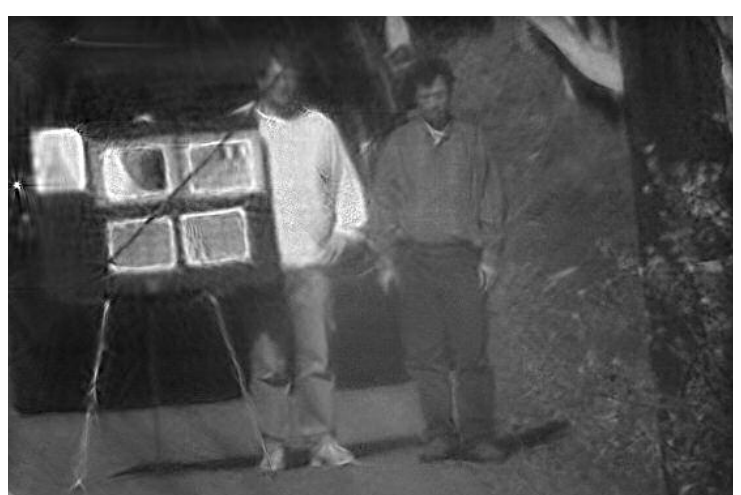

3b. Speckle processed result from 100 raw frames

Figure 3: Personnel imaged at a $3.3 \mathrm{~km}$ (2.0 mile) distance using a $15 \mathrm{~ms}$ exposure time.

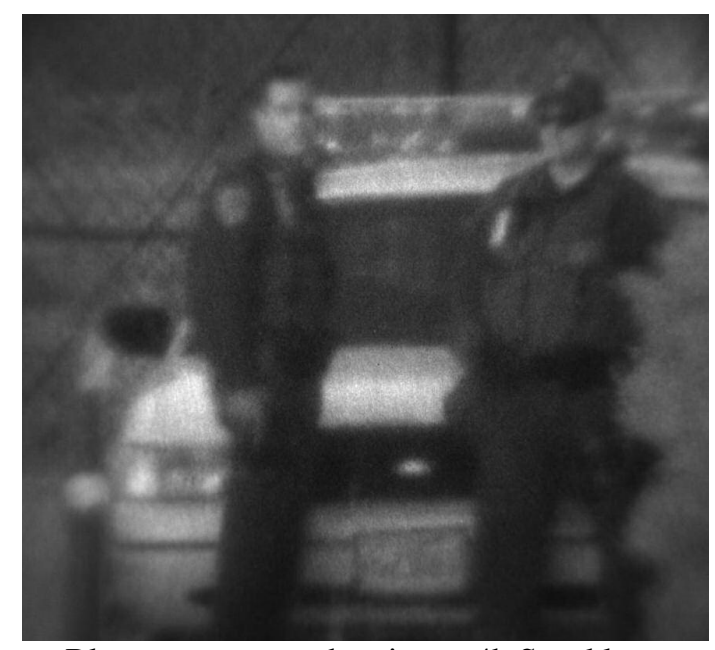

4a: Blurry, unprocessed raw image

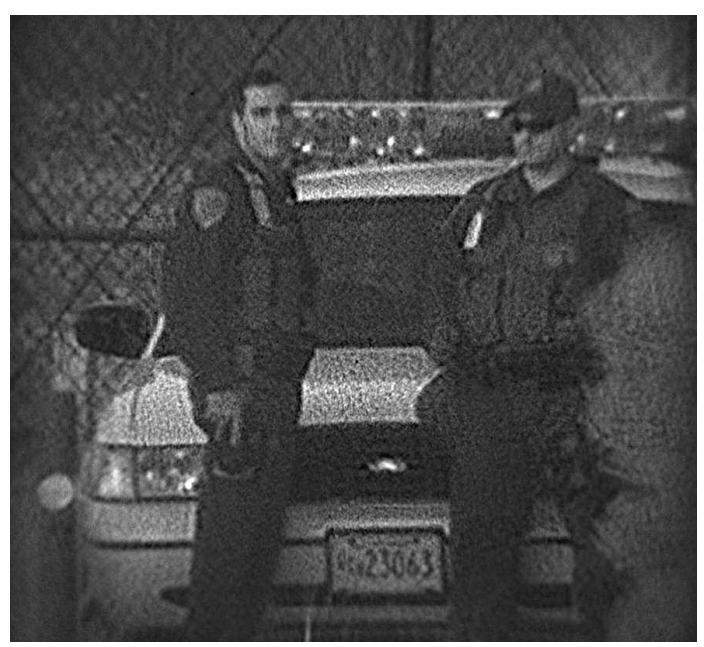

4b. Speckle processed result from 100 raw frames

Figure 4: Personnel imaged at a $1.5 \mathrm{~km}$ distance at sunset with image intensification using a $10 \mathrm{~ms}$ exposure time. 


\subsubsection{Stationary imaging of vehicles}

This next experiment was performed in order to determine experimentally if sub-field speckle imaging would be applicable in improving long slant-range surveillance images of vehicles. The imaging was performed from the top of Mt. Diablo, a 3849' peak located approximately 30 miles east of San Francisco. The location was selected to demonstrate surveillance performance from a high hillside vantage point as well as what could be expected from a low flying UAV. The weather was cool, and the visibility, hence the image contrast, was less than optimal due to a surface haze layer approximately 1000 feet thick. We staged the vehicle experiment with three different types of vehicles: a 20 foot long flatbed truck, a 24 foot long water truck, and a 23.5 foot long cargo van.

Results of the vehicles at $22 \mathrm{~km}$ range are displayed in Figure 5. A sample frame is shown in Figure 5a and the speckle processed result is shown in $5 \mathrm{~b}$. The processed result clearly shows three distinct types of vehicles. The rightmost truck is identifiable as a flatbed. Also, the fence posts along the road and between the fields become clearly visible.

The next result, displayed in Figure 6, is of the same vehicles parked at a range of $37 \mathrm{~km}$. In the sample frame, it is not obvious what we are looking at, but in the processed image, we can see that there are three vehicles on what appears to be a road. Likewise, the foliage looks much sharper and crisper in the processed image.

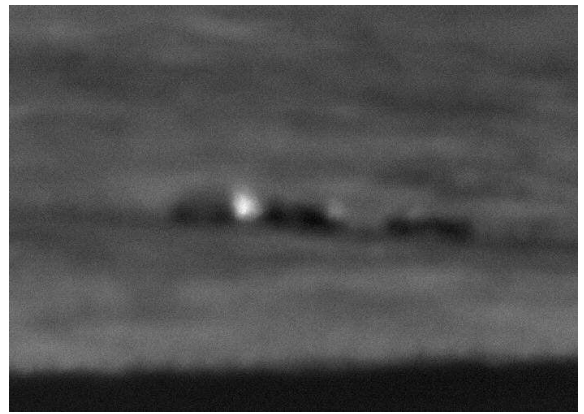

5a: Blurry, unprocessed raw image

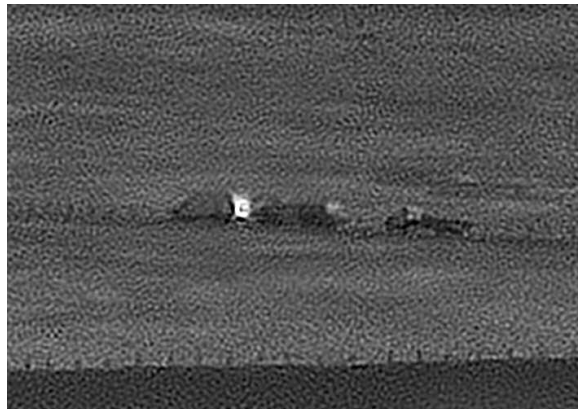

5b. Speckle processed result from 100 raw frames

Figure 5: Vehicles imaged at a $22 \mathrm{~km}$ (14 mile) distance through a Celestron 8-inch aperture telescope with a monochrome CCD camera using a 1 millisecond exposure time.

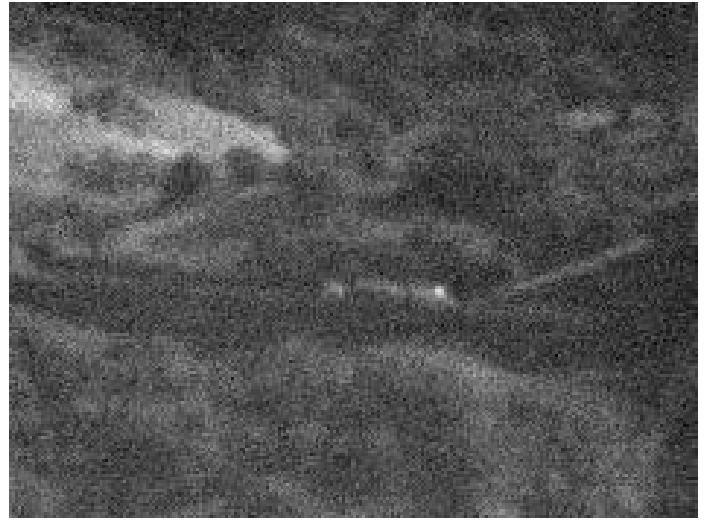

6a: Blurry, unprocessed raw image

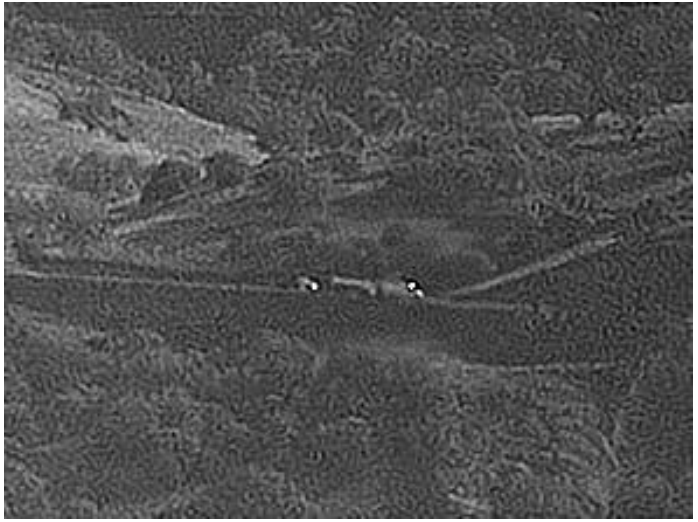

6b. Speckle processed result from 100 raw frames

Figure 6: Same three trucks at $37 \mathrm{~km}$ range. The leftmost vehicle is the flatbed truck, the center vehicle is the water truck, and the rightmost vehicle is the cargo van. 


\section{ADAPTATIONS FOR NON-STATIONARY IMAGERY}

For simplification purposes, let us consider the case of an object such as a vehicle moving through the field-of-view of a stationary imaging system. The primary problem for the speckle imaging algorithm will be the relative motion of the vehicle with respect to its background. We have simulated a sequence of frames of a moving car with atmospheric blurring corresponding to $\mathrm{D} / \mathrm{r}_{0}=10$. We extract out a sub-image centered on the car from each frame. Figures $7 \mathrm{a}$ and $7 \mathrm{~b}$ are the first and last images in the sequence. Figure $7 \mathrm{c}$ shows the result of speckle processing applied to this sequence. Because of the changing background in each frame, the reconstructed image of the car is dominated by artifacts.

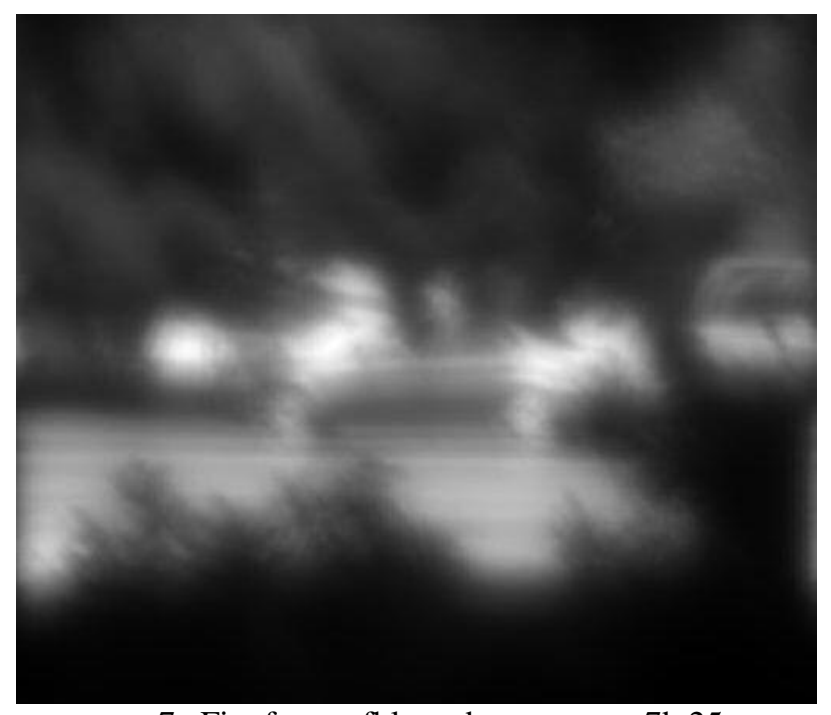

7a. First frame of blurred car sequence

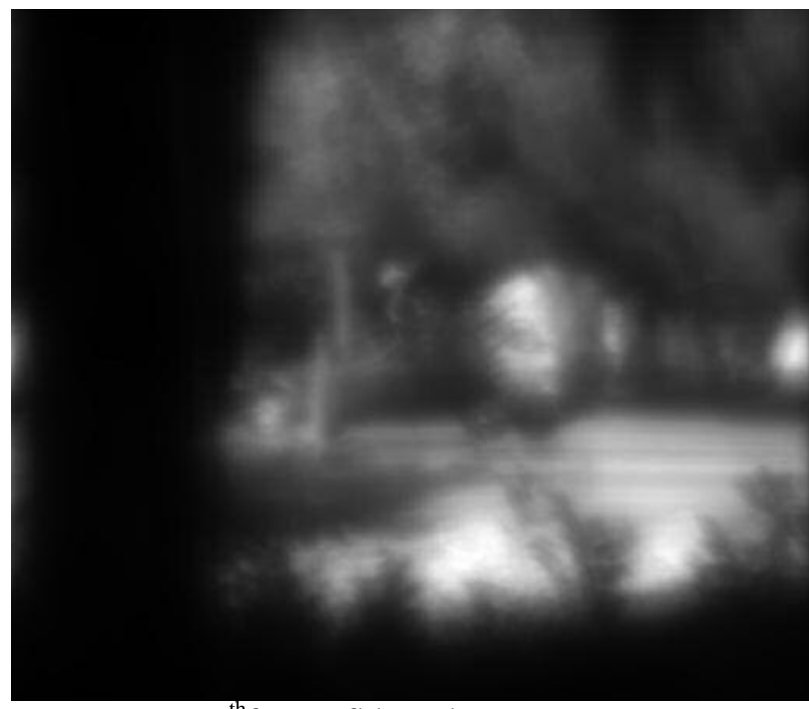

7b. $25^{\text {th }}$ frame of blurred car sequence

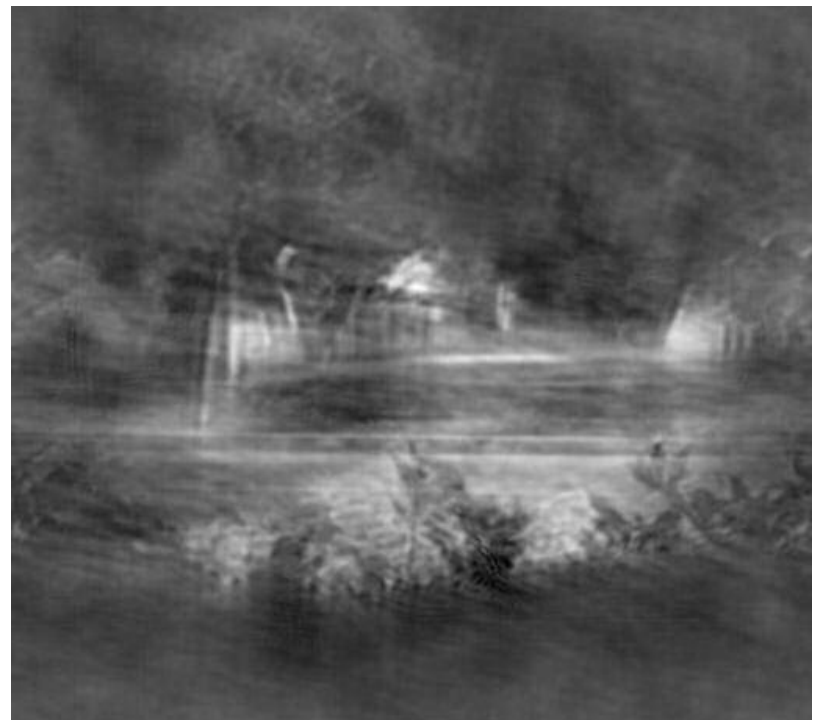

7c. Speckle processed result - where's the car?

Figure 7. Example of what happens when attempting to speckle process image frames with relative target to background motion. In this case the car was held in the center of the frame and the background allowed to change. 


\subsection{Pre-processing method}

From looking at the result in Figure 7, it is clear that something must be done to remove the background in order to enhance the vehicle. We have found it very effective to apply a tapered window around the object of interest in each frame once the object has been centered in each frame. The window shape needs to conform roughly to the object shape. We have found that a $3^{\text {rd }}$ order super-Gaussian rectangular window surrounding the object is sufficient for the vehicle cases examined thus far. In Figure $8 \mathrm{a}$ and $8 \mathrm{~b}$ we show two windowed image frames, and in Figure $8 \mathrm{c}$, we see the dramatically improved speckle reconstruction after the changing background foliage in each frame has been removed. The image processing steps are summarized in Figure 9.

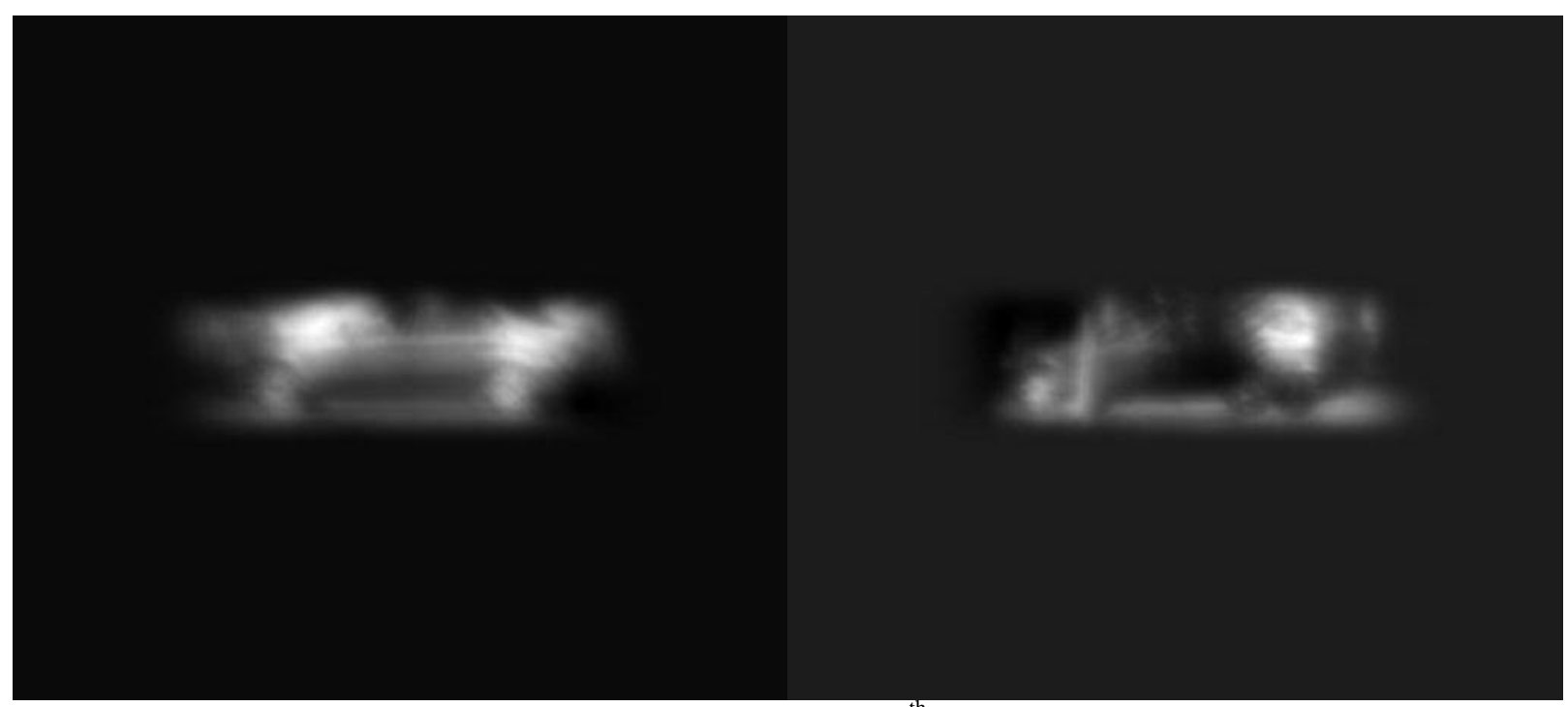

8a. First frame of blurred car sequence, windowed

8 b. $25^{\text {th }}$ frame of blurred car sequence, windowed

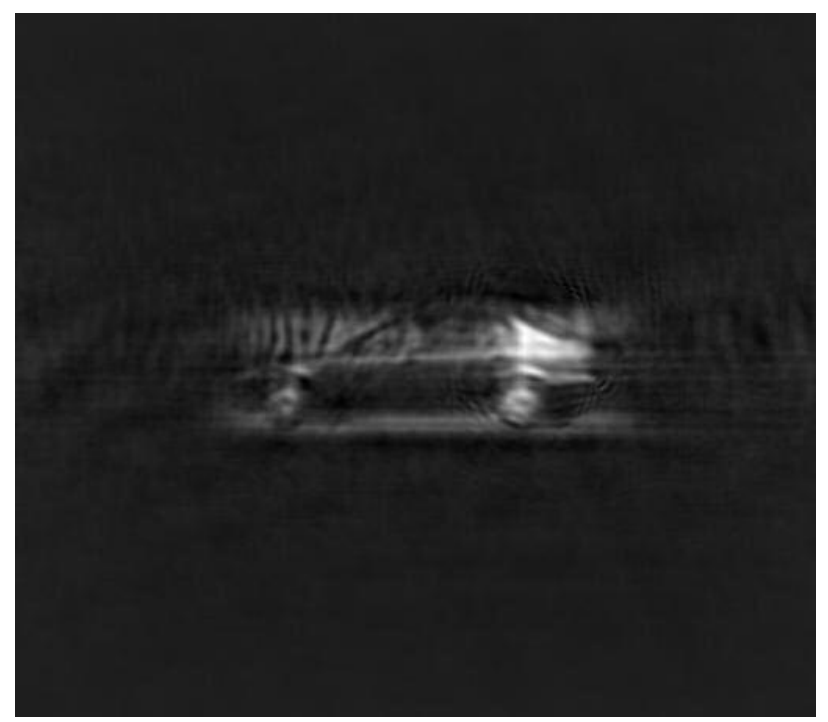

8c. High-resolution speckle processed result

Figure 8: Example result showing the effectiveness of windowing to remove the moving background scenery prior to speckle processing. 


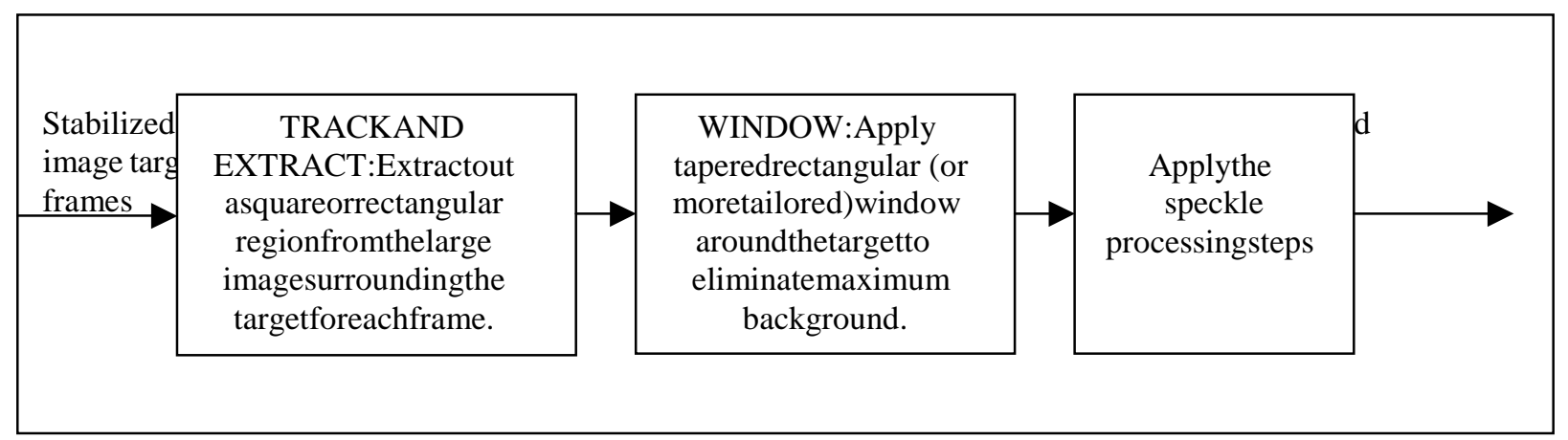

Figure 9: Image processing steps for enhancing multi-frame imagery with relative target to background motion.

\subsection{Experimental results}

The imagery in Figure 10 was acquired from the top of Mt. Diablo at a range of approximately $13 \mathrm{~km}$ away. The camera was operating at video rate ( $\sim 34$ FPS) with an exposure time of 1 millisecond and thus able to capture vehicles traveling at highway speeds for a sufficient number of frames before exiting the field of view. Again, the optics employed were an 8 inch diameter telescope with a 2 meter focal length. Since the camera pixel size is $7.4 \mu \mathrm{m}$ square and images at a wavelength of $0.5 \mu \mathrm{m}$, we are undersampling the diffraction limit by a factor of 3 . Nevertheless, significant atmospheric blurring is evident.

We decided to enhance the large semi-truck, as shown highlighted in Figure 10. The first step of the image pre-processing is to perform target tracking and extraction. We show sample frames in Figure 11. Next, we apply an appropriately sized window around the target in each frame. Sample windowed frames are displayed in Figure 12. The data sequence is then ready for bispectral reconstruction. For this particular case, $128 \times 128$ pixel sized tiles were used, and an $r_{0}$ of $7.5 \mathrm{~mm}$ was used in the processing. The much higher resolution speckle processed image is shown in Figure 13. Greater details about the cab structure are visible and more detail regarding the truck type is available in the processed image. For comparison, in Figure 14, we show what happens when the registered and windowed frames are simply added together. The result is a smooth and less noisy image, but still at low resolution.

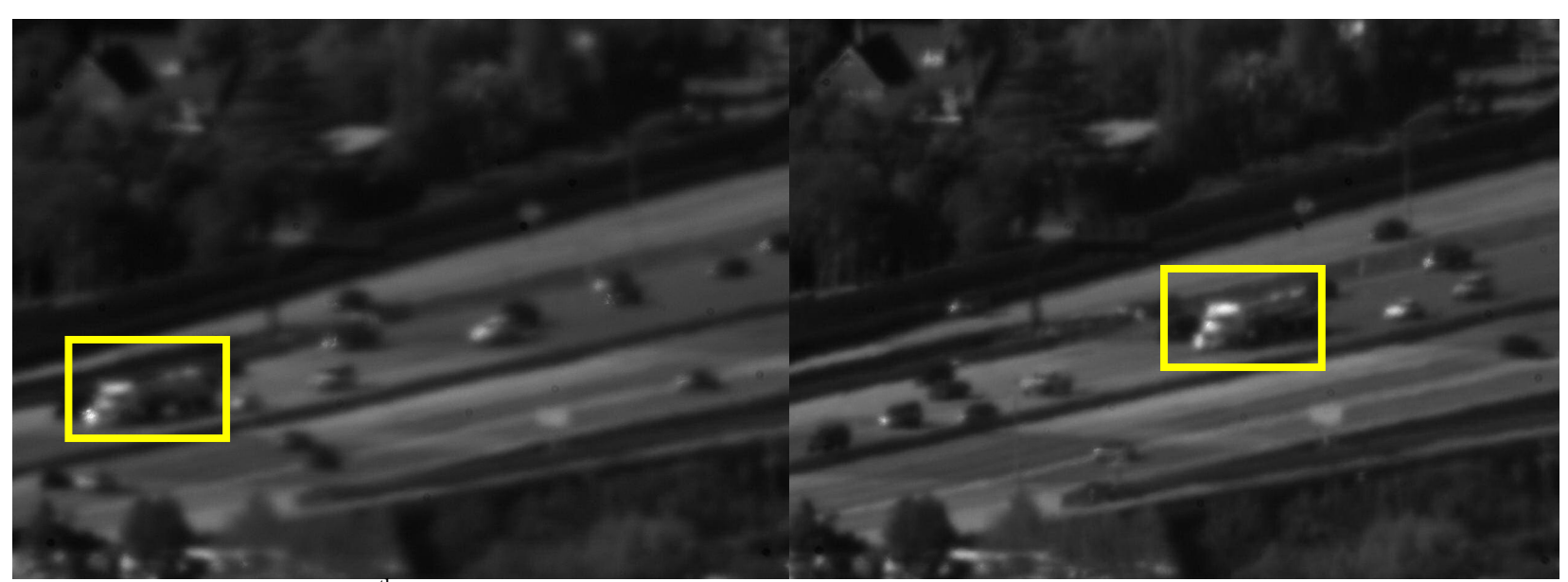

Figure 10: First and last $\left(75^{\text {th }}\right)$ raw image frames of vehicles on a highway from 7 miles away.

Image scale is $25 \%$ of full size. 


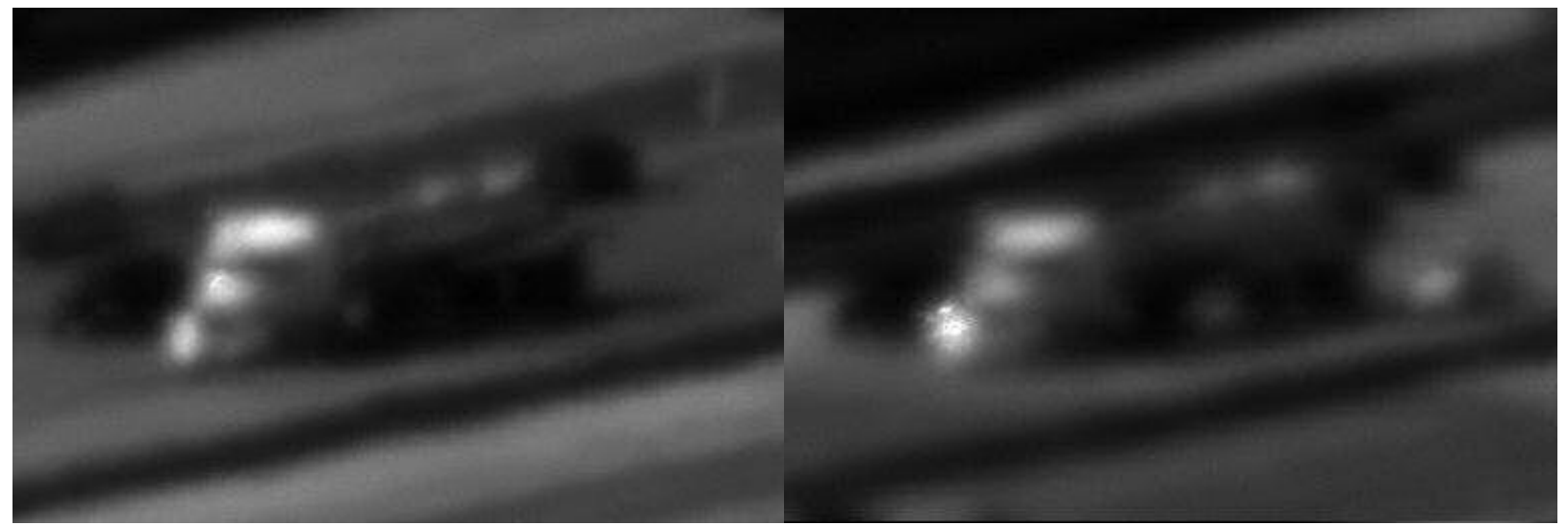

Figure 11: First and last frame of image sequence where the truck has been tracked and extracted out. Image scale is $80 \%$ of full size. The extracted image size is $384 \times 256$ pixels.

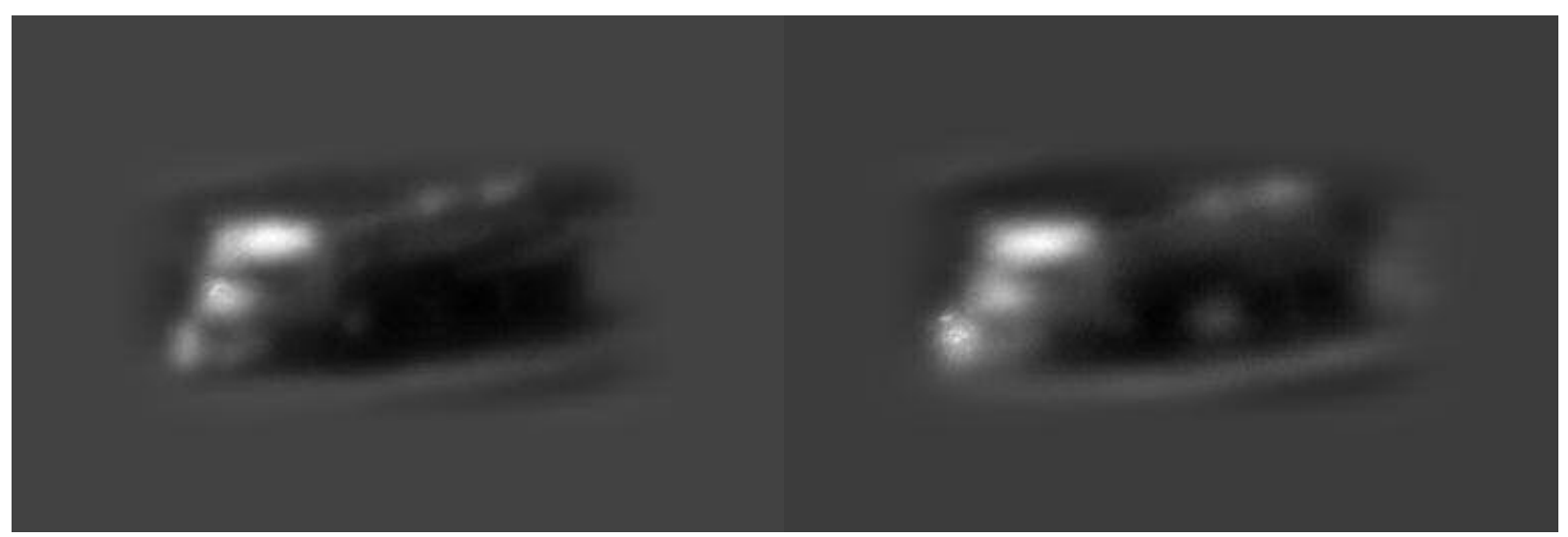

Figure 12: First and last frame of image sequence after target tracking, extraction, and windowing.

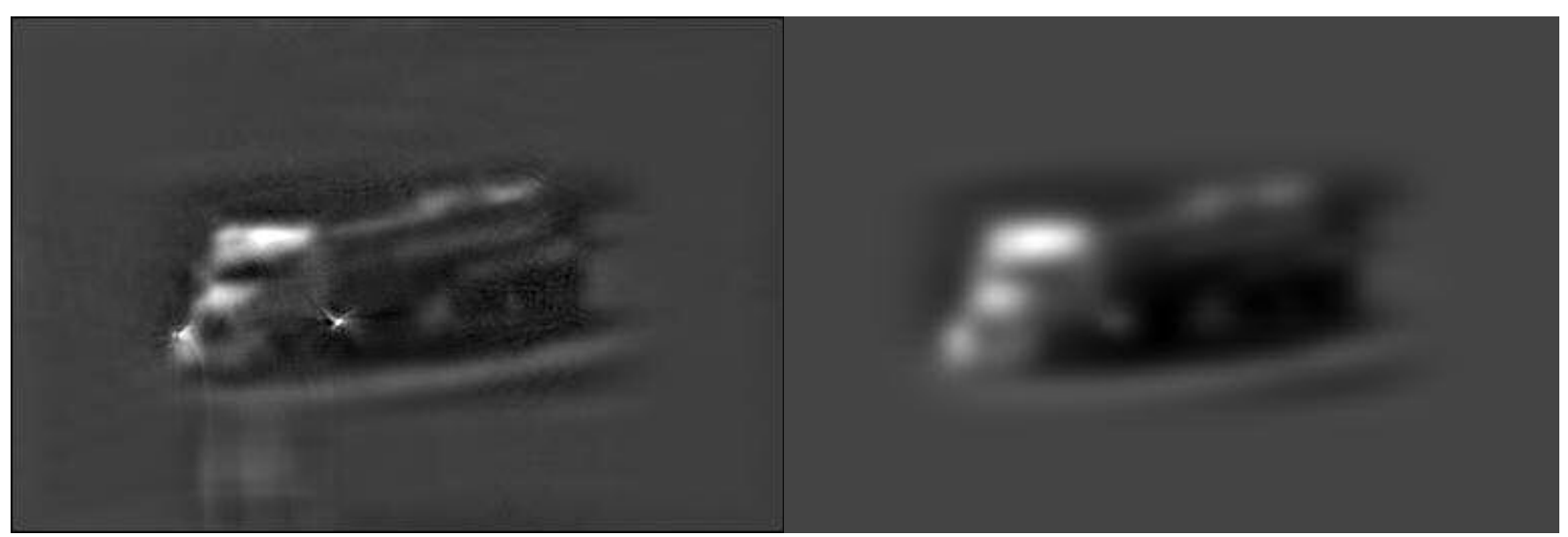

Figure 13: High-resolution speckle processed result

Figure 14: Simple addition of the registered frames 
During the same experiment, we captured a few frames of a passing helicopter from about 15-20 km away. Only 14 frames were available for processing. We show the first and last "tracked and extracted" sub-frames in Figure $15 \mathrm{a}$ and $\mathrm{b}$. The higher resolution speckle-processed result after windowing around the helicopter is shown in Figure $15 \mathrm{c}$.

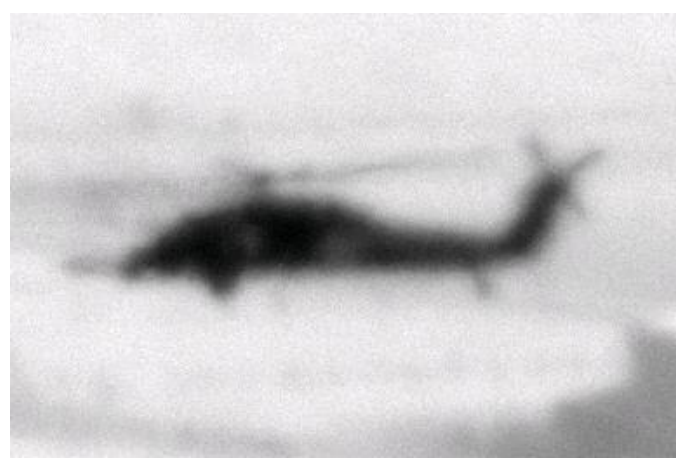

15a. First tracked and extracted sub-frame

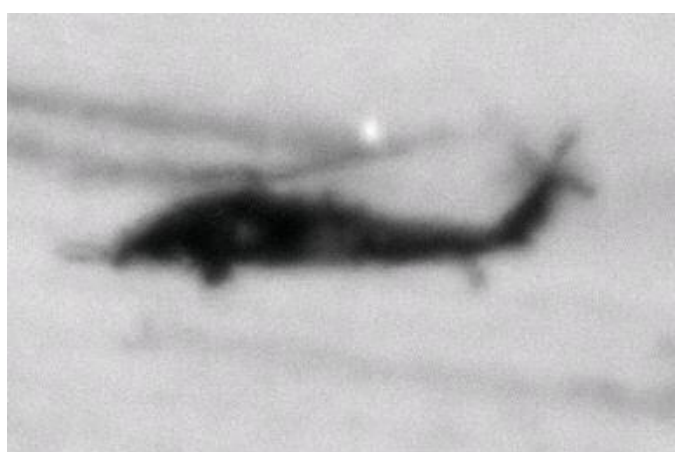

15b. $14^{\text {th }}$ track and extracted sub-frame

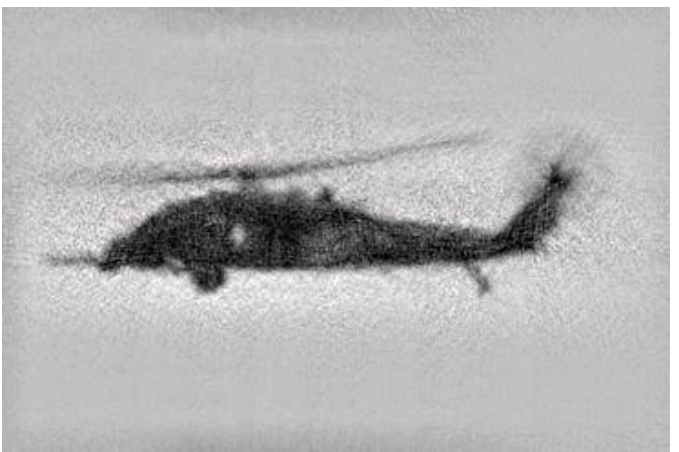

15c. Higher-resolution speckle processed result

\subsection{Additional considerations and limitations}

A primary limitation of speckle imaging of moving targets will be related to the velocity of the target. When the target is moving too fast, it is possible that the image of the target will be fundamentally blurred within the exposure time. By using fast exposure times on the order of a millisecond, it is possible to successfully image vehicles traveling at highway speeds. Another limitation imposed by the vehicle traveling too fast is that the object may move out of the camera field of view before acquiring a sufficient number of frames if the camera is not actively tracking the target. A minimum sufficient number of frames depends on factors such as signal to noise ratio and the amount of atmospheric blurring, but is typically 20 to 30 . The plot in Figure 16 illustrates the dependence between object velocity and the number of frames that are obtainable with an example imaging system.

For the experimental case considered in section 3.2, the optical system was 3 times undersampled, the CCD size was $1600 \times 1200$ pixels, and the target was at $13 \mathrm{~km}$ range. Interpolating and scaling the numbers in the plot, and assuming a $60 \mathrm{mph}$ velocity we could expect to obtain more than 100 frames of the target. (i.e. 20 frames * 1600 pixels/1000 pixels $* 3 * 34 \mathrm{FPS} / 30 \mathrm{FPS}=109$ frames). This is consistent with our observations.

\section{CONCLUSIONS}

We have demonstrated high-resolution imaging using speckle-imaging techniques not only for static targets and platforms, but for the case when there is relative target to background motion. This capability is potentially important for imaging and identification of certain types of tactical targets which are moving with respect to the imaging system. 


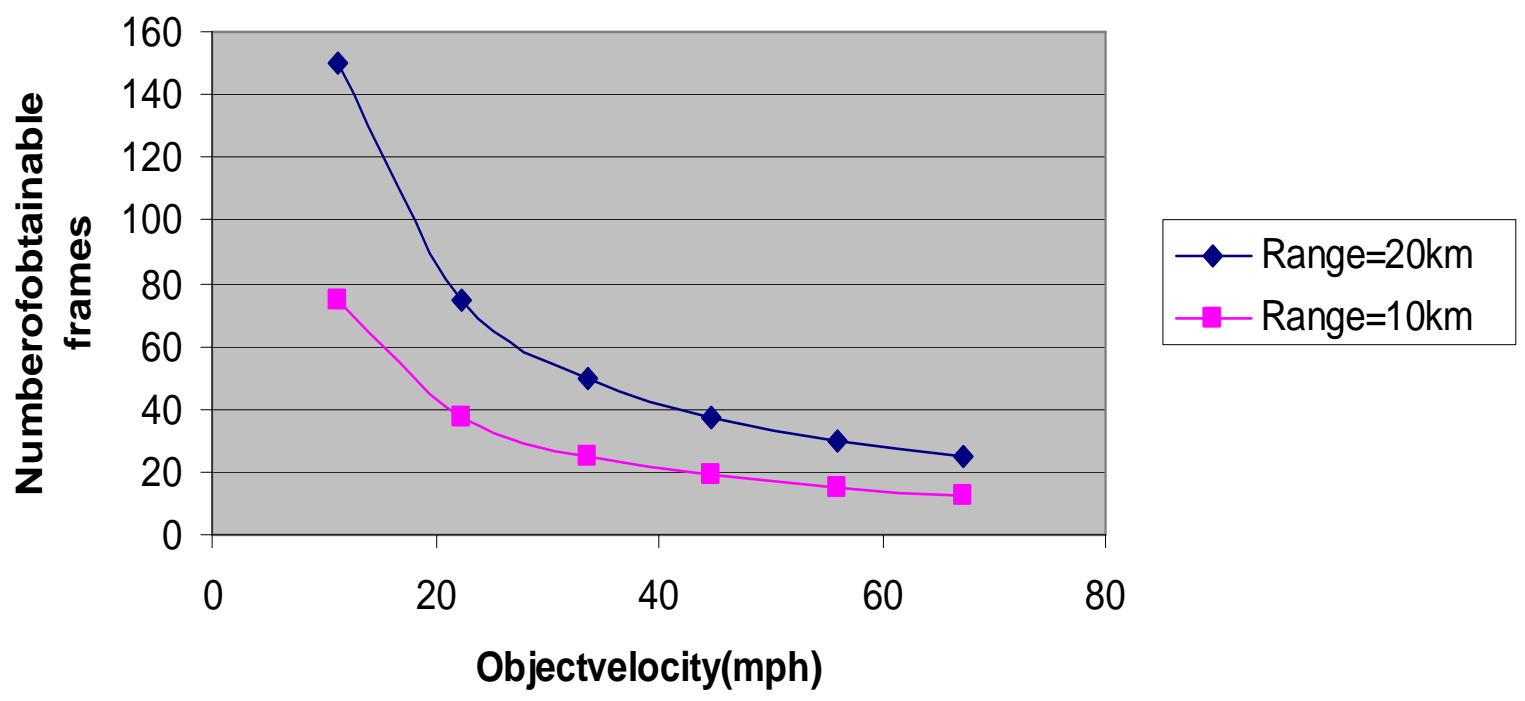

Figure 16: Plot of the number of obtainable frames vs. object velocity for a target range of $10 \mathrm{~km}$ and $20 \mathrm{~km}$. This assumes the imaging system is Nyquist sampling the aperture $(\mathrm{D}=20 \mathrm{~cm}, \lambda=0.5 \mu \mathrm{m})$, and using a 1000x1000 pixel sized CCD that is sampling at video-rate (30 FPS).

\section{ACKNOWLEDGEMENTS}

I would like to acknowledge the help of Doug Poland and Mike Newman for the moving vehicle experiments performed from Mt. Diablo.

Work performed under the auspices of the U.S. Department of Energy by the UC Lawrence Livermore National Laboratory under contract No. W-7405-ENG-48.

\section{REFERENCES}

1. C. J. Carrano, "Speckle Imaging over Horizontal Paths", Proceedings of the SPIE -High Resolution Wavefront Control: Methods, Devices, and Applications IV, 4825, 109-120, (2002)

2. C. J. Carrano, J. M. Brase, "Horizontal and Slant Path Surveillance with Speckle Imaging", AMOS Technical Conference Proceedings, (2002)

3. C. J. Carrano, "Progress in horizontal and slant-path imaging using speckle imaging", Proceedings of the SPIELASE2003 - Optical Engineering at the Lawrence Livermore National Laboratory, 5001 (2003) pp. 56-64

4. D. L. Fried, "Optical Resolution through a Randomly Inhomogeneous Medium for Very Long and Very Short Exposures", JOSA, Vol 56, No. 10, 1372-1379 (1966)

5. T. W. Lawrence, J.P. Fitch, D. M. Goodman, N. A. Massie, R. J. Sherwood, and E. M. Johansson, "Extended-image reconstruction through horizontal path turbulence using bispectral speckle interferometry," Opt. Eng. 32, 627-636 (1992) 